\title{
SOME MORE RESULTS ON A GENERALIZED 'USEFUL' R-NORM INFORMATION MEASURE
}

\author{
SATISH KUMAR
}

Abstract. A parametric mean length is defined as the quantity

$$
{ }_{R \beta} L_{u}=\frac{R}{R-1}\left[1-\sum P_{i}^{\beta}\left(\frac{u_{i}}{\sum u_{i} p_{i}^{\beta}}\right)^{\frac{1}{R}} D^{-n_{i}\left(\frac{R-1}{R}\right)}\right],
$$

where $R>0(\neq 1), \sum p_{i}=1$. This being the useful mean length of code words weighted by utilities, $u_{i}$. Lower and upper bounds for $R_{\beta} L_{u}$ are derived in terms of 'useful'-R-norm information measure for the incomplete power distribution, $p^{\beta}$.

\section{Introduction}

Consider the following model for a random experiment $S$,

$$
S_{N}=[E ; P ; U]
$$

where $E=\left(E_{1}, E_{2}, \ldots, E_{N}\right)$ is a finite system of events happening with respective probabilities $P=\left(P_{1}, P_{2}, \ldots, P_{N}\right), p_{i} \geq 0$, and $\sum p_{i}=1$ and credited with utilities $U=\left(u_{1}, u_{2}, \ldots, u_{N}\right), u_{i}>0, i=1,2, \ldots, N$. Denote the model by $E$, where

$$
E=\left[\begin{array}{cccc}
E_{1} & E_{2} & \ldots & E_{N} \\
p_{1} & p_{2} & \ldots & p_{N} \\
u_{1} & u_{2} & \ldots & u_{N}
\end{array}\right]
$$

We call (1.1) a Utility Information Scheme (UIS). Belis and Guiasu [3] proposed a measure of information called 'useful information' for this scheme, given by

$$
H(U ; P)=-\sum u_{i} p_{i} \log p_{i}
$$

Received December 18, 2007; revised November 25, 2008.

Key words and phrases. R-norm entropy, 'useful' R-norm information, utilities, power probabilities. 
where $H(U ; P)$ reduces to Shannon's [9] entropy when the utility aspect of the scheme is ignored i.e., when $u_{i}=1$ for each $i$. Throughout the paper, $\sum$ will stand for $\sum_{i=1}^{N}$ unless otherwise stated and logarithms are taken to base $D(D>1)$.

Guiasu and Picard [5] considered the problem of encoding the outcomes in (1.1) by means of a prefix code with codewords $w_{1}, w_{2}, \ldots, w_{N}$ having lengths $n_{1}, n_{2}, \ldots, n_{N}$ and satisfying Kraft's inequality [4].

$$
\sum_{i=1}^{N} D^{-n_{i}} \leq 1
$$

Where $D$ is the size of the code alphabet. The useful mean length $L_{u}$ of code was defined as

$$
L_{u}=\frac{\sum u_{i} n_{i} p_{i}}{\sum u_{i} p_{i}}
$$

and the authors obtained bounds for it in terms of $H(U ; P)$. Longo [7], Gurdial and Pessoa [6], Autar and Khan [1], Singh and Rajeev [8], have studied generalized coding theorems by considering different generalized measures of (1.2) and (1.4) under condition (1.3) of unique decipherability.

In this paper, we study some coding theorems by considering a new function depending on the parameters $R$ and $\beta$ and a utility function. Our motivation for studying this new function is that it generalizes 'useful' R-norm information measure already existing in the paper Singh and Rajeev [8], Bockee and Lubbe [2].

\section{Coding Theorems}

In this section, we define 'useful' R-norm information measure as:

$$
{ }_{R \beta} H(U ; P)=\frac{R}{R-1}\left[1-\left(\frac{\sum u_{i} p_{i}^{R \beta}}{\sum u_{i} p_{i}^{\beta}}\right)^{\frac{1}{R}}\right],
$$

where $R>0(\neq 1), \beta>0, p_{i} \geq 0, i=1,2, \ldots, N$ and $\sum p_{i}=1$.

(i) When $\beta=1$ then (2.1) reduces to 'useful' R-norm information measure studied by Singh and Rajeev [8].

$$
\text { i.e. }{ }_{R} H(U ; P)=\frac{R}{R-1}\left[1-\left(\frac{\sum u_{i} p_{i}^{R}}{\sum u_{i} p_{i}}\right)^{\frac{1}{R}}\right] \text {. }
$$

(ii) When $u_{1}=1$ and $\beta=1$, (2.1) reduces to R-norm entropy as considered by Bockee and Lubbe [2].

$$
\text { i.e. }{ }_{R} H(P)=\frac{R}{R-1}\left[1-\left(\sum p_{i}^{R}\right)^{\frac{1}{R}}\right] \text {. }
$$

(iii) When $\beta=1$ and $R \rightarrow 1$, (2.1) reduces to a measure of 'useful' information for the incomplete distribution due to Belis and Guiasu [3]. 
(iv) When $u_{i}=1$ for each $i$, i.e. when the utility aspect is ignored, $\sum p_{i}=1, \beta=1$ and $R \rightarrow 1$, the measure (2.1) reduces to Shannon's entropy [9].

$$
\text { i.e. } H(P)=-\sum p_{i} \log p_{i} \text {. }
$$

(v) When $u_{i}=1$ for each $i$, the measure (2.1) becomes R-norm entropy for the $\beta$ power distribution derived from $P$. We call ${ }_{R \beta} H(U ; P)$ in $(2.1)$ the generalized 'useful' R-norm information measure for the incomplete power distribution $P^{\beta}$.

\section{Further consider}

Definition. The 'useful' mean length ${ }_{R \beta} L_{u}$ with respect to 'useful' R-norm information measure is defined as :

$$
{ }_{R \beta} L_{u}=\frac{R}{R-1}\left[1-\sum p_{i}^{\beta}\left(\frac{u_{i}}{\sum u_{i} p_{i}^{\beta}}\right)^{\frac{1}{R}} D^{-n_{i}\left(\frac{R-1}{R}\right)}\right],
$$

where $R>0(\neq 1), \sum p_{i}=1$.

(i) For $\beta=1$ and $R \rightarrow 1,{ }_{R \beta} L_{u}$ in (2.5) reduces to the useful mean length $L_{u}$ of the code given in (1.4)

(ii) For $\beta=1, u_{i}=1$ for each $i$ and $R \rightarrow 1,{ }_{R \beta} L_{u}$ becomes the optimal code length defined by Shannon [9].

(iii) For $\beta=1, u_{i}=1$ then (2.5) reduced to ${ }_{R} L$ considered by Bockee and Lubbe [2].

$$
\text { i.e. }{ }_{R} L=\frac{R}{R-1}\left[1-\sum p_{i} D^{-n_{i}\left(\frac{R-1}{R}\right)}\right] \text {, }
$$

the average length of code word considered by Bockee and Lubbe [2].

We establish a result, that in a sense, provides a characterization of ${ }_{R \beta} H(U ; P)$ under the condition of unique decipherability.

Theorem 2.1. For all integers $D>1$

$$
{ }_{R \beta} L_{u} \geq_{R \beta} H(U ; P)
$$

under the condition (1.3). Equality holds if and only if

$$
n_{i}=-\log _{D}\left(\frac{u_{i} P_{i}^{R \beta}}{\sum u_{i} p_{i}^{R \beta}}\right) .
$$

Proof. We use Holder's [10] inequality

$$
\sum x_{i} y_{i} \geq\left(\sum x_{i}^{p}\right)^{\frac{1}{p}}\left(\sum y_{i}^{q}\right)^{\frac{1}{q}}
$$


for all $x_{i} \geq 0, y_{i} \geq 0, i=1,2, \ldots, N$ when $P<1(\neq 1)$ and $p^{-1}+q^{-1}=1$, with equality if and only if there exists a positive number $c$ such that

$$
x_{i}^{p}=c y_{i}^{q} .
$$

Setting

$$
\begin{aligned}
& x_{i}=p_{i}^{\frac{R \beta}{R-1}}\left(\frac{u_{i}}{\sum u_{i} p_{i}^{\beta}}\right)^{\frac{1}{R-1}} D^{-n_{i}}, \\
& y_{i}=p_{i}^{\frac{R \beta}{1-R}}\left(\frac{u_{i}}{\sum u_{i} p_{i}^{\beta}}\right)^{\frac{1}{1-R}},
\end{aligned}
$$

$p=1-\frac{1}{R}$ and $q=1-R$ in (2.9) and using (1.3) we obtain the result (2.7) after simplification for $\frac{R}{R-1}$ as $R>1$.

Theorem 2.2. For every code with lengths $\left\{n_{i}\right\}, i=1,2, \ldots, N,{ }_{R \beta} L_{u}$ can be made to satisfy,

$$
{ }_{R \beta} L_{u}<_{R \beta} H(U ; P) D^{\left(\frac{1-R}{R}\right)}+\frac{R}{R-1}\left[1-D^{\left(\frac{1-R}{R}\right)}\right] .
$$

Proof. Let $n_{i}$ be the positive integer satisfying, the inequality

$$
-\log _{D}\left(\frac{u_{i} p_{i}^{R \beta}}{\sum u_{i} p_{i}^{R \beta}}\right) \leq n_{i}<-\log _{D}\left(\frac{u_{i} p_{i}^{R \beta}}{\sum u_{i} p_{i}^{R \beta}}\right)+1 .
$$

Consider the intervals

$$
\delta_{i}=\left[-\log _{D}\left(\frac{u_{i} P_{i}^{R \beta}}{\sum u_{i} P_{i}^{R \beta}}\right),-\log _{D}\left(\frac{u_{i} P_{i}^{R \beta}}{\sum u_{i} P_{i}^{R \beta}}\right)+1\right]
$$

of length 1 . In every $\delta_{i}$, there lies exactly one positive number $n_{i}$ such that

$$
0<-\log _{D}\left(\frac{u_{i} P_{i}^{R \beta}}{\sum u_{i} p_{i}^{R \beta}}\right) \leq n_{i}<-\log _{D}\left(\frac{u_{i} P_{i}^{R \beta}}{\sum u_{i} p_{i}^{R \beta}}\right)+1 .
$$

It can be shown that the sequence $\left\{n_{i}\right\}, i=1,2, \ldots, N$ thus defined, satisfies (1.3). From (2.14) we have

$$
\begin{aligned}
& n_{i}<-\log _{D}\left(\frac{u_{i} P_{i}^{R \beta}}{\sum u_{i} P_{i}^{R \beta}}\right)+1 \\
& \Rightarrow D^{-n_{i}}>\left(\frac{u_{i} P_{i}^{R \beta}}{\sum u_{i} P_{i}^{R \beta}}\right) D^{-1} \\
& \Rightarrow D^{-n_{i}\left(\frac{R-1}{R}\right)}>\left(\frac{u_{i} P_{i}^{R \beta}}{\sum u_{i} p_{i}^{R \beta}}\right)^{\frac{R-1}{R}} D^{\frac{1-R}{R}} .
\end{aligned}
$$


Multiplying both sides of $(2.15)$ by $p_{i}^{\beta}\left(\frac{u_{i}}{\sum u_{i} p_{i}^{\beta}}\right)^{\frac{1}{R}}$, summing over $i=1,2, \ldots, N$ and simplification for $\frac{R}{R-1}>0$ as $R>1$, gives (2.11).

Theorem 2.3. For every code with lengths $\left\{n_{i}\right\}, i=1,2, \ldots, N$, of Theorem 2.1, ${ }_{R \beta} L_{u}$ can be made to satisfy,

$$
{ }_{R \beta} L_{u} \geq{ }_{R \beta} H(U ; P)>{ }_{R \beta} H(U ; P) D+\frac{R}{R-1}(1-D) .
$$

Proof. Suppose

$$
\bar{n}_{i}=-\log _{D}\left(\frac{u_{i} P_{i}^{R \beta}}{\sum u_{i} p_{i}^{R \beta}}\right) .
$$

Clearly $\bar{n}_{i}$ and $\bar{n}_{i}+1$ satisfy 'equality' in Holder's inequality (2.9). Moreover, $\bar{n}_{i}$ satisfies Kraft's inequality (1.3).

Suppose $n_{i}$ is the unique integer between $\bar{n}_{i}$ and $\bar{n}_{i}+1$, then obviously $n_{i}$, satisfied (1.3).

Since $R>0(\neq 1)$, we have

$$
\begin{aligned}
\sum p_{i}^{\beta}\left(\frac{u_{i}}{\sum u_{i} p_{i}^{\beta}}\right)^{\frac{1}{R}} D^{-n_{i} \frac{(R-1)}{R}} & \leq \sum p_{i}^{\beta}\left(\frac{u_{i}}{\sum u_{i} p_{i}^{\beta}}\right)^{\frac{1}{R}} D^{-\bar{n}_{i} \frac{(R-1)}{R}} \\
& <D\left(\sum p_{i}^{\beta}\left(\frac{u_{i}}{\sum u_{i} p_{i}^{\beta}}\right)^{\frac{1}{R}} D^{-\bar{n}_{i} \frac{(R-1)}{R}}\right) .
\end{aligned}
$$

Since,

$$
\sum p_{i}^{\beta}\left(\frac{u_{i}}{\sum u_{i} p_{i}^{\beta}}\right)^{\frac{1}{R}} D^{-\bar{n}_{i}} \frac{(R-1)}{R}=\left(\frac{\sum u_{i} p_{i}^{R \beta}}{\sum u_{i} p_{i}^{\beta}}\right)^{\frac{1}{R}} .
$$

Hence, (2.18) becomes

$$
\sum p_{i}^{\beta}\left(\frac{u_{i}}{\sum u_{i} p_{i}^{\beta}}\right)^{\frac{1}{R}} D^{-n_{i} \frac{(R-1)}{R}} \leq\left(\frac{\sum u_{i} p_{i}^{R \beta}}{\sum u_{i} p_{i}^{\beta}}\right)^{\frac{1}{R}}<D\left(\frac{\sum u_{i} p_{i}^{R \beta}}{\sum u_{i} p_{i}^{\beta}}\right)^{\frac{1}{R}},
$$

which gives the result (2.16).

\section{References}

[1] R. Autar and A. B. Khan, On generalized useful information for incomplete distribution, J. of Comb. Information and Syst. Sci. 14(1989), 187-191.

[2] E. Bockee and J. C. A. Vander Lubbe, The R-norm information measure, Information and Control 45(1980), 136-155.

[3] M. Belis and S. Guiasu, A qualitative-quantitative measure of information in cybernetics systems, IEEE Trans. Information Theory, IT -14(1968), 593-594. 
[4] A. Feinstein, Foundation of Information Theory, McGraw Hill, New York, 1958.

[5] S. Guiasu and C. F. Picard, Borne infericutre de la longuerur utile de certain codes, C.R. Acad. Sci, Paris 273A(1971), 248-251.

[6] Gurdial and F. Pessoa, On useful information of order $\alpha$, J. Comb. Information and Syst. Sci. 2(1977), 158-162.

[7] G. Longo, A noiseless coding theorem for sources having utilities, SIAM J. Appl. Math. 30(4)(1976), 732-738.

[8] R. K. Tuteja, R. P. Singh and Rajeev Kumar, Application of Holder's Inequality in information theory, Information Sciences 152(2003), 145-154.

[9] C. E. Shannon, A Mathematical Theory of Communication, Bell System Tech-J. 27(1948), 394-423, 623-656.

[10] O. Shisha, Inequalities, Academic Press, New York, 1967.

Department of Mathematics, Faculty of Education, Mekelle University, P.O.Box 3050, Mekelle, Ethiopia.

E-mail: drsatish74@rediffmail.com 